\title{
PSICOSSOCIOLOGIA APLICADA AO ESPORTE: CONTRIBUIÇÃO PARA A SUA COMPREENSÃO
}

\author{
Antonio Carlos SIMÕES" \\ Dante De ROSE JUNIOR**
}

\section{INTRODUÇÃO}

O presente texto é produto de um pensar em Esporte, colocando perspectivas para se compreender a natureza das manifestações humanas com relação a tudo aquilo que se qualifica/quantifica em favor da performance. $O$ Esporte se auto denomina como um modelo de realidade social, no qual se desenvolve a vida dos desportistas e onde aparecem e se diferenciam os mais e/ou menos qualificados - aponta, embora de diferentes formas, os vários caminhos para os indivíduos. $\dot{E}$ constituído, pois, por elementos culturais, sociais, filosóficos, ideológicos, econômicos, políticos e psicológicos. O Esporte tem um sentido amplo incluindo modalidades individuais/coletivas e que escapam ao controle dos que dele participam. Põe em destaque um amplo repertório de conhecimento ao seu dinamismo social.

O Esporte é muito mais complexo do que se pensa. A participação individual, em grupo $\mathrm{e}$ institucional envolve na realidade, um julgamento de valor em relação aos comportamentos, formas de agir e de reagir dentro do cenário dos diferentes tipos de esportes. Distingamos que forças sociais de influências do poder maior, em relação ao menor, podem assumir ou representar fenômenos que se manifestam por via dos indivíduos, equipes, clubes, entre outros elementos constitutivos do esporte moderno; coloca os conceitos nos caminhos das articulações explicitadas das infra-estruturas do Esporte com os fenômenos que os condicionam, com os contextos e ambientes em que se desenvolvem o intercâmbio de comportamento e as manifestaç̃es em todas as situações.

Uma análise é feita mostrando os problemas das realidades humanas em função das intervenções que devem ser efetuadas - sob forma de interesses, necessidades e de tensões que penetram e ajudam a tecer a rede dos fenômenos psicossociais dentro e fora dos grandes e pequenos grupos sociais esportivos. Entenda-se, que quando se trabalha com os diferentes esportes individuais e coletivos, as investigações ganham em amplitude e flexibilidade, refletindo que fenômenos psicossociais estão dentro de uma macro e microsituação, o que significa que no universo esportivo, as necessidades, percepções, opiniões, sentimentos, reações, influências, entre outras, propiciam causas e efeitos nas performances de condutas no plano da participação e liberdade individual, as quais procuramos conhecer através da psicossociologia do esporte.

\section{A PROBlemática do CONHECIMENTO PSICOSSOCIAL}

A preocupação com o conhecimento do Esporte não é nova. Quando o Esporte chega à presença dos diferentes segmentos sociais descreve todo um conjunto de manifestações humanas que fundamentam as discussões sobre conceitos e

\footnotetext{
- Coordenador do Laboratório de Psicossociologia do Esporte da Escola de Educação Física e Esporte da Universidade de São Paulo.

** Coordenador do Grupo de Estudos e Pesquisas em Psicologia do Esporte da Escola de Educação Física e Esporte da Universidade de São Paulo.
} 
definições que o caracterizam como fenômeno e/ou instituição social. Quaisquer que sejam as percepções, existe o risco de se confundir o pensamento científico com os conceitos para a escolha dos instrumentos de análises e de pesquisas. E isso se manifesta, de fato, na vida dos profissionais/estudiosos tanto nas áreas da Educação Física/Esporte quanto de outras áreas afins. O Esporte parece ter conhecimento simples, quando considerado do ponto de vista da percepção individual. Neste caso, os conceitos e a forma de se observar e descrever com objetividade os fatos esportivos estão impregnados de pensamentos restritos às diferentes formas de sua interpretação.

As posições características assumidas ante esses objetivos tem acarretado uma multiplicidade de conceitos, sobretudo das formas de conhecimento das ciências humanas (antropologia, filosofia, sociologia, psicologia), sobre a explicação dos fatos a serem pesquisados, descritos e da observação dos fenômenos, que atualmente vão além do senso comum esportivo. $O$ Esporte se torna assim, um modelo de conhecimento complexo, um saber interligado com outros domínios - um conhecimento específico, imediato e direto enquanto pensar. Ele cria um complexo de conhecimento difuso e universal diante do seu papel social e da sua representação objetiva ou subjetiva na vida dos indivíduos. Por conseguinte, a validade das análises sociológicas e psicológicas provêm de níveis conceituais muito diferentes.

O cabedal de conhecimento acadêmico ou popular torna-se visão de uma realidade diferenciada com a impossibilidade de fundamentar conceitos em relação ao senso esportivo comum. Este conhecimento é constituído entre as formas de explicações de uns e de outros, que encontram nas práticas esportivas uma oportunidade de conceituar a heterogeneidade dos acontecimentos. Acima de tudo, são as teorias, por um lado, e a realidade social e psicológica por outro, que dão dentro de um quadro referencial, as linhas de pensamento e tendências para dar uma visão à realidade dos fenômenos no campo do esporte moderno. As manifestações humanas/esportivas, segundo Vuori \& Fentem (1996), tem como objetivo a expressão das competências atlética, social e psicológica que se inserem num vasto continuum de conceitos positivos ou negativos. Seus meios de comunicação são a televisão, rádio, jornal, revistas e livros textos especializados em diferentes tipos de esportes individuais e coletivos. Tais publicações fazem do Esporte um modelo riquíssimo de crenças populares e de demandas à realidade das performances de condutas de dirigentes, técnicos e atletas em competições nacionais e internacionais.

Este problema se coloca de forma mais objetiva, na questão da relação "indivíduo/ciência/tecnologia" - relação válida dentro de um sistema de produtividade, onde as proezas refletem um avanço em direção às performances visíveis. Em termos gerais, o esporte possui um perfil próprio, envolvendo os indivíduos dentro de um contexto que se define como sendo natural de manifestações humanas, no qual se desenvolve a vida esportiva dos indivíduos, onde "todos" se consideram "atletas" - e no sentido mais amplo por atividades que se impõem ao bem-estar em competir. Assim, por exemplo tudo aquilo que é social/esportivo integra-se ao cultural - torna-se popular e divulgado pelos meios de comunicação de massa.

Ibáñez (citado por González, 1997) considerou aos meios de comunicação como "dispositivos privilegiados de produção, circulação e consumo de verdades" - sobretudo com as emoções e com os sentimentos dos indivíduos dentro e fora dos locais dos jogos. É condicionante, dentro das possibilidades oferecidas ou impostas aos indivíduos em vislumbrar o Esporte como um modelo de realidade social, em relação ao qual as instituições, clubes, equipes e indivíduos são os grandes representantes deste cenário criando um "estado- espetáculo" com a totalidade de suas capacidades interativas e funcionais.

$O$ poder do Esporte, então, leva os indivíduos a certas conseqüências no que se refere às manifestações populares. $\mathrm{O}$ fenômeno mental da conduta popular transforma dirigentes, técnicos e atletas em heróis, mitos e modelos representativos de comportamentos (Eurico Miranda, Luiz Felipe, Edmundo, Marcelinho Carioca, Romário) desvendando como representação do real, a cultura das diferentes modalidades esportivas individuais e coletivas. Esta visão exige não só conhecimentos gerais de Esporte, mas nas contingências da sua representação nas crenças populares, e isso faz com que o Esporte seja mais observado e menos interpretado. Trata-se, de um modelo social que transpõe os conceitos e problemas psicossociais característicos dos esportes aquáticos e terrestres em todos os segmentos sociais e dentro das instituições. Essas repercussões são de natureza diversa no sentido de acentuar a 
relacionados às condições interativas e funcionais, que não podem ser reduzidas a mera formalidades. A relatividade restrita dos fenômenos dá conta e expressa as condições de convivência e de sobrevivência de dirigentes, técnicos e atletas neste cenário.

Ninguém tem a noção exata da natureza, extensão e profundidade dos impactos dos fenômenos sociológicos e psicológicos sobre 0 comportamento dos indivíduos no Esporte, que se organizam em função do custo/beneficio e com as lideranças econômicas e políticas que promovem 0 espetáculo esportivo. É nele que se inserem as tendências externas dos poderes sociais, e internamente, por uma série de elementos que, orientam técnicos e atletas nas direções mais inesperadas - e que condicionam esses personagens e suas manifestações em torno da confirmação histórica do "Homem Esportivo" considerações que nos leva ao campo psicossocial propriamente dito.

\section{A PSICOSSOCIOLOGIA E A SUA LEGITIMIDADE ESPECÍFICA NO ESPORTE}

Em relação à sociedade, o esporte moderno levou os pesquisadores/estudiosos a concebê-lo como uma realidade altamente competitiva semelhante a de um modelo que implica, conforme Freitas (1993, p.26)

na existência de corporações que organizam e quantificam a relação custo/benefício nos investimentos feitos, transformando 0 esporte em um produto mercadológico, com estratégias planejadas...(sic)

Com isso, o viés interpretativo sobre as instituições, equipes e indivíduos está nos diferentes caminhos conceituais (científicos, tecnológicos) que são definidos pelas diferentes culturas para explicitar os problemas existentes no Esporte. Quando vistos como um conjunto de forças impulsoras e/ou limitantes, os conceitos inseridos no processo de racionalização (qualificação, quantificação) nos levam as inúmeras situações que temos que percorrer obrigatoriamente, a dicotomia que vai do "Homo Ludens" ao "Homo Faber", tendo em vista a substituição do Homem que participava (lúdico) em atividades esportivas e jogos ao Homem da ciência e da tecnologia, diferenciando assim, as brincadeiras e jogos do esporte de alta performance.

O conceito singular predominante do Esporte enquanto movimento foi substituído, segundo Heinilã (1990), por conceitos múltiplos da cultura do corpo, das atividades físicas e das práticas esportivas competitivas. A psicossociologia do esporte, como todas as disciplinas empiricas, acumula conhecimentos básicos e específicos referentes à relatividade e determinação dos conhecimentos básicos aplicados ao Esporte. A maneira de entender varia segundo o campo das atividades relacionadas com os padrões definidos de organização, métodos de procedimentos de trabalhos empregados, comportamentos indicativos de amizade, confiança mútua e interatividade ligada à maior ou menor ênfase colocada no campo do individual ou do coletivo.

Como um campo de conhecimento, a psicossociologia do Esporte têm recebido contribuições de profissionais de diferentes áreas de conhecimento (antropologia, sociologia, psicologia), mas com visões estruturadas, por vezes altamente preconcebidas a respeito do que significa trabalhar no contexto do Esporte de "performance" - portanto, com conceitos teóricos e práticos restritos às suas próprias formações profissionais. Não se trata de um campo de conhecimento único, mas em relação aos problemas envoltos com aspectos psicossociais vinculados com 0 movimento humano/manifestações esportivas. Nesse ponto de vista é que se pode acentuar a validade e a importância dos estudos e pesquisas em psicossociologia do Esporte; quão importante quanto a antropologia cultural e biológica, fisiologia, pedagogia e filosofia.

Os estudos voltados para as questões de racionalização, discriminação, inclusão e exclusão dos mais e menos habilidosos são essenciais em sua dimensão quantitativa, para a transformação do conhecimento esportivo e de seus objetivos segundo uma linha mais científica - na transposição de tudo aquilo que é lúdico, participativo e competitivo. Isto sugere que a apreensão da realidade psicossocial esportiva é complexa e diferencia-se das que predominam em outras áreas pela explícita ligação do macro e do microscópico das questões investigadas com os problemas do mundo dos esportes de performance - e por uma forte interdependência dos aspectos sociais e psicológicos com o comportamento das grandes e pequenas instituições/equipes esportivas. Algumas, coin os acontecimentos observáveis à 
comportamentos, sentimentos, expectativas de ascensão social, motivos de agir e reagir, e outras com as relações para explicar o individual e coletivo.

Muchielli (1978, p.12), escreveu que a realidade psicossocial é específica - e

que o maior erro seria o de acreditar que um fenômeno social seja a soma de pequenos fenômenos individuais...(sic).

A isso acrescentam-se as idéias de que os conceitos devem satisfazer certas exigências metodológicas aplicadas a um cenário esportivo, que possui uma realidade específica em sua relação social e psicológica. O grau de ligação entre as intervenções de dirigentes, técnicos, atletas e performances de condutas pessoais tem importância fundamental quando se deseja fazer predições para especificar comportamentos associados a um universo qualitativo e quantitativo dentro dos estádios, ginásios, piscinas e pistas de corridas.

$O$ que torna esse quadro mais complexo está ligado a um conjunto de fenômenos psicossociais que moldam a coerência orgânica e funcional às definições dos valores predominantes na relação custo/beneficio que não excluem, mas reforçam o papel dos dirigentes, técnicos e atletas enquanto fortes aliados na consecução de seus objetivos; basta dizer que a temática racional da competição decorre da capacidade de rendimento às condições individuais e ao desempenho coletivo das equipes. Não é mera coincidência que "quase ou todos" os fenômenos interativos e de execução de tarefas estariam ligados a um repertório de condutas individuais e coletivas como promotoras do "estado espetáculo" Este é, pois, um cenário intrincado de interações sociais entre os indivíduos e os diferentes tipos de espetáculos esportivos. $\mathrm{Na}$ verdade, o esporte enquanto um modelo de realidade social se impõe aos indivíduos, exerce influências. É neste nível que o comportamento, segundo Della Torres (1986) é extremamente complexo por seus motivos aprovativos (medalhas, prêmios, elogios, ascensão) e punitivos ( derrotas, vaias, desprestígio), que não podem ser decompostos senão para análise.

Os recursos à formação e desenvolvimento de vínculos institucionais, grupais e individuais são complexos e envolvem mais do que entender do arrematar ao gol, encestar, bloquear, cortar e/ou conquistar vitórias - qualquer análise antecipada não nos permite predizer o comportamento esportivo. É preciso observar que os comportamentos e influências interpessoais vão se integrando no cotidiano das instituições/clubes e das equipes esportivas - seus comportamentos conjuntos, opiniões, sentimentos, ações e reações são sensíveis e criam fatos na maneira de pensar, sentir e agir de qualquer indivíduo. Quer dizer que o individual está colocado com o coletivo; 0 individual e coletivo colocados com o institucional; e 0 individual coletivo e institucional colocados com as realidades e irrealidades fenomenológicas da sociedade - portanto - caminham numa direção vincular entre o macro e o micro-sistema social, conforme demonstrado pela FIGURA 1.

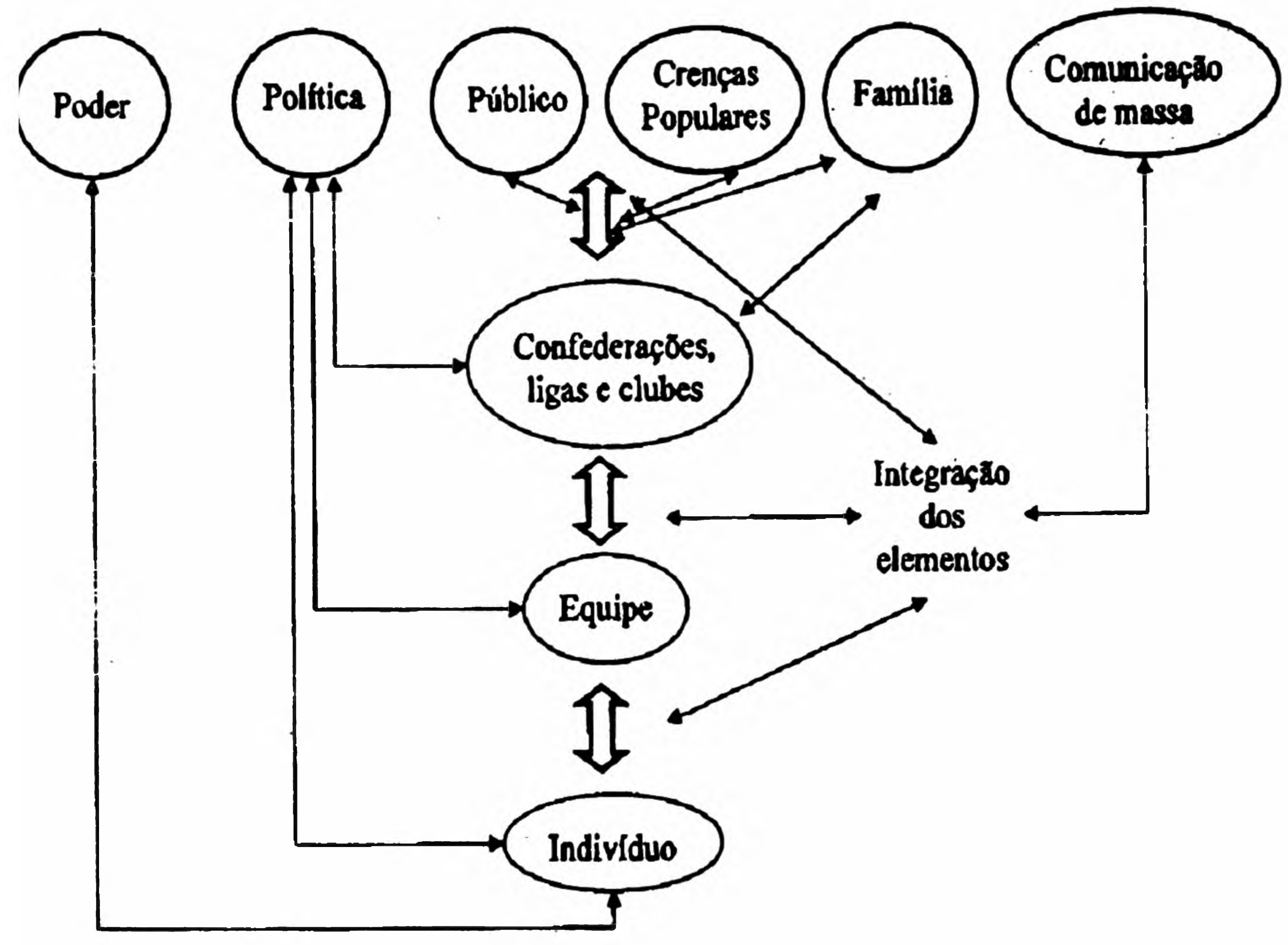

O macro-sociológico o microsociopsicológico no esporte têm suas fontes de influências nos próprios mecanismos orgânicos e funcionais do esporte de "performance" - e são realidades fenomenológicas definíveis que ficam sempre interdependentes e indissociáveis entre os determinismos relativos ligados ao poder maior em relação ao menor e/ou do poder menor para o maior.

FIGURA 1 - Dimensões das relações macro-sociológicas e micro-sociopsicológicas. (Adaptado de Chappuis \& Thomas, 1988). 
Não há dúvidas que o sistema de relações mantido entre o macro e o micro-sistema esportivo comporta uma idéia predominantemente orientada para os aspectos orgânicos e funcionais das ações decisivas de objetividade. É um sistema que assume simultânea e sucessivamente um grau de coerência social e operativa entre as forças propulsoras e forças limitadoras da participação individual/coletiva. Elas são imperativas aos critérios adotados, passando do homogêneo ao heterogêneo, do estrutural ao funcional $e$ do individual ao coletivo. Os fenômenos psicossociais estariam presentes num contexto contínuo de influencias mútuas entre os grandes e os pequenos grupos sociais esportivos. Teríamos, assim, relações de aproximação e/ou de afastamentos como rivalidades dos indivíduos entre si, com o grupo ou com as instituições das quais eles fazem parte, cujos fatos vão pautar o comportamento dos dirigentes, técnicos e atletas dentro dos grandes e pequenos grupos sociais esportivos.

O problema central, portanto, é o sistema das relações institucionais, grupais e individuais, no que concerne a formação, desenvolvimento e formas de cooperação entre 0 macro e micro-sistema esportivo e, finalmente, das dependências daí resultantes. É claro que com essa caracterização a idéia de que os fenômenos psicossociais existem nas conexões entre os indivíduos, grupos e instituições esportivas estão presentes e influenciando a vida cotidiana dos indivíduos no mundo dos esportes. Daí os estudos dos mais complicados problemas de intercâmbio de comportamentos em situações que se caracterizam por um alto grau de indefinição entre dirigentes, técnicos, atletas e de outros profissionais que convivem e sobrevivem no cenário esportivo.

O Esporte cria, segundo Bracht (1989), um mundo dentro do mundo, sobretudo quando relacionado com ações desafiadoras. O que faz uma equipe ser melhor que a outra? Por que a performance de conduta pessoal de um técnico não é tão boa quanto a de outros? O que ocorre com os valores individuais dentro das equipes? $O$ que ocorre com o "Ideal próprio" dos técnicos quando os atletas atribuem a eles perfis autocráticos, participativos ou democráticos? O que os atletas pensam de seus técnicos? Técnicos e atletas "despersonalizam" valores individuais em prol da coletividade, e, em que direção? O que ocorre com os valores individuais quando são testados pelo meio ambiente em que treịnam e jogam? Qual é o comportamento ideológico de liderança dos técnicos no esporte de rendimento?

Cada instituição/clube, equipe, indivíduo é mais do que se supõe que seja (e as vezes menos) do que se espera; condicionado pelo contexto das relações sociais e funcionais que traz, em si mesmo, a idéia de competir e de vencer a "qualquer" custo. A forte institucionalização das manifestações esportivas é resultante da evolução dos conceitos científicos em direção aos elementos envolvidos com o comportamento humano no esporte de "performance" Não é preciso imaginação para ver as conseqüências sociais e psicológicas na relação dos indivíduos que promovem o "estado espetáculo" Guareschi et alii (1995) escreveram que os indivíduos adquirem e desenvolvem suas identidades pessoais, criando símbolos e interagindo-se com os demais. As relações interpessoais e as funções operacionais aparecem com grande intensidade com o meio esportivo, responsável pela ascensão social tanto dos dirigentes/técnicos quanto dos atletas, sendo considerados como elementos determinantes do bem-estar humano nos clubes e equipes.

Não se pode esquecer, porém, que esses elementos incluem a riqueza dos relacionamentos, o nível de infra-estrutura e seu entorno, como as condições concebidas pelos indivíduos em consonância ou dissonância a si próprios, com os outros em sentido sociopsicológico. Por exemplo, 0 indivíduo apreende-se a si mesmo em uma função específica quando está jogando, e outro quando se relaciona com os dirigentes, técnicos e companheiros. Em ambos os casos, o "Eu" observa o "Eu" que observa o "Eu" dos outros, e o "Eu" dos outros, que observa o seu próprio "Eu" perante os outros. Além disso, mostra um quadro relativamente estável que se contempla numa perspectiva única de ocupação espacial e individual de rendimento.

A teoria "Lewiana" fundamenta que as ações dos indivíduos estão estruturadas num campo de forças, cujas intervenções dependem, em grande parte, da própria estabilidade desse campo. $\mathrm{O}$ poder das forças sociais individuais e coletivas estão interligadas para assegurar as influências e discussões sobre os problemas enfrentados. As manifestações humanas, em tudo associadas à dinâmica das relações sociais e funcionais, refletem um comportamento de acordo com os seus interesses - e mantido por "performances" de condutas pessoais altamente competitivas no mundo dos esportes. Elas são delicadas nos 
diferentes tipos de esportes individuais e coletivos - realidades fenomenológicas, organizativas e perceptivas, que Antonelli \& Salvini (1978), postularam depender das funções que são exigidas e das interações que se tenham.

\section{DAS CONCEPÇÕES TEÓRICAS ÀS PESQUISAS PSICOSSOCIAIS NO ESPORTE}

As pesquisas psicossociais, segundo Mucchieli (1978), trazem em si a compreensão sobre os fenômenos envolvidos com a eficácia dos diversos organismos sociais. Isso se deve principalmente aos conceitos relacionados com as Ciências do Esporte e com a multiplicidade do conhecimento em Ciências Humanas. Compreender os pressupostos que balizam cada investigação e validar esse conhecimento corresponde, de forma objetiva, às indagações mais prementes no cenário do Esporte de performance. A filosofia, à sociologia, e à psicologia se impõem tarefas de grande magnitude. As idéias surgem, tornando-se claro que a compreensão dos problemas esportivos estão principalmente relacionados em nível filosófico e psicossocial. Isto foi reforçado pelas concepções filosóficas que definem o esporte como a atividade humana que supera em universalidade 0 poder das forças sociais, políticas, ideológicas, econômicas, religiosidades e diferentes tipos de "performance" de condutas pessoais.

A filosofia do Esporte baseia seus estudos, segundo Perez (1991), na realidade sociológica para conceituar e definir uma gama de evidências e de situações que podem estar expostas, em relação às atividades humanas. $\mathrm{O}$ repertório de comportamentos dessas atividades é fruto de uma longa e contínua evolução das manifestações esportivas. A eficiência e/ou ineficiência com que os indivíduos lidam com 0 mundo esportivo se mede pela variabilidade e adaptação de seus comportamentos - e qualquer tentativa de prognóstico ou diagnóstico envolve, imprescindivelmente, estudos que incidem nos indivíduos, nos grupos, nas instituições e nas relações desses elementos. Nesse caminho, todo enfoque é uma espécie de concepção filosófica relacionada com a integração e interdisciplinaridade dos fatores envolvidos com a evolução histórica-filosófica do esporte, como a axiologia, praxeologia e caráter social de seus fundamentos; natureza psicossocial e ecológica dos gêneros das ações motrizes; natureza sócio-cultural das atividades ludomotoras; raizes sociológicas/antropológicas da praxeologia e com as necessidades de contextualizar os objetos (institucionalização), normas que tornam dificeis a posição dos indivíduos na instituição e reflexão na universalidade dos elementos, na política e na sociedade, acerca dos objetivos que definem as propriedades formais do conhecimento do Esporte. Enfim, com social, filosófico, religioso; científico e tecnológico num "continuum" valioso de acontecimentos no mundo dos esportes aquáticos $\mathrm{e}$ terrestres. Chega-se, então, à compreensão de que a escolha dos objetos de estudos no esporte devem ser determinados no âmbito de discussões mais profundas, e de procedimentos metodológicos condizentes com a realidade do esporte de performance. Com isso, as pesquisas nas áreas da sociologia e a psicologia do esporte investigam o mundo esportivo em que os indivíduos vivem e sobrevivem. Tanto uma, como a outra, recorre à observação e às análises que faz sobre os problemas sociais e psicológicos enfrentados pelos indivíduos dentro dos grandes e pequenos grupos sociais. Ambas contam com uma curta história na maioria dos países, tendo no Journal of Sport Psychology e na International Review for the Sociology of Sport, a base da publicação dos estudos e pesquisas do Esporte. Exemplo disso, é que Heinemann \& Preuss (1990), publicaram uma heterogeneidade de trabalhos relacionados com os aspectos de socialização, violência, agressão, entre outros. O mesmo ocorrendo com a psicologia do esporte, que obteve seu reconhecimento internacional com a publicação de trabalhos de revisão sobre a investigação e aplicação de instrumentos, especialmente, com Martens (1979), Salmela (1979), Hanin (1980), que proporcionaram, segundo Riera \& Cruz (1990) os primeiros trabalhos sobre a personalidade dos desportistas - preparação psicológica - para aumentar suas capacidades de rendimento nas competições.

Não há dúvidas de que a conexão da sociologia com a psicologia no esporte fez com que fosse criado pelo Departamento de Esporte da Escola de Educação Física e Esporte da Universidade de São Paulo, o Laboratório de Psicossociologia do Esporte - 0 primeiro laboratório até onde sabemos, que busca conhecimento em relação ao dinamismo do Esporte moderno. O "LAPSE" é um dos laboratórios que faz parte do Centro de Excelência do "INDESP" e tem como objetivo o Estudo do Esporte, Indivíduos e Grupos considerados do ponto de vista sistêmico, relacionados às seguintes áreas da 
Ciência do Esporte: Antropologia (biológica/cultural), Filosofia, Sociologia, Psicologia e Esporte de Performance. Diriamos que essas áreas são potencialmente significativas para os estudos do Esporte moderno, tendo em vista que podem ser relacionadas, de forma substantiva e não arbitrárias no campo evolutivo do Esporte incluindo a teoria da evolução como um poderoso referencial para se falar do Comportamento Humano no Esporte.

A proposição mais concreta dessa teoria, segundo Nicholson (1998), está relacionada com as necessidades e anseios muito variados entre o sucesso e insucesso dos indivíduos. Eles arriscam e estruturam inúmeras situações que retratam a realidade competitiva em termos de comportamento - onde cometer erros envolve perdas (possivelmente de seus empregos), e jogar "limpo" representa enfrentar a difícil e ingrata tarefa de estruturar desafios. As pesquisas são decisivas e mostram que, apesar das interações e a capacidade de trabalhar em equipe, os individuos preferem ser compreendidos a compreender e se posicionam com opiniões para tomar decisões e em assumir a responsabilidade por elas. Os estudos e pesquisas desenvolvidos pelo "LAPSE" são movidos a desafios, em resultados que contribuem decisivamente para o desenvolvimento de trabalhos nos grandes e pequenos grupos esportivos. Esses estudos e pesquisas promovem o ensinamento de novos conceitos sobre o esporte de "performance" e auxilia os profissionais da Área do Esporte a tomar decisões e trabalhar em equipe.

O "LAPSE" tem investido em equipamentos, cujo funcionamento e utilidade são de conhecimento dos grupos de estudos, alunos de graduação e pós-graduação. Os dados obtidos com as pesquisas são apresentados em eventos nacionais e internacionais, e utilíssimos nas devolutivas para os técnicos, atletas e dirigentes. Os encontros são, sobretudo, de trabalhos voltados para a vida dos indivíduos no mundo dos esportes individuais e coletivos. Para isso, os Grupos de estudos e pesquisas trabalham com procedimentos (linhas de pesquisas) para se definir prática e dinamicamente os eventos comportamentais no esportes, que certamente facilitam responder a uma lista de razões que justifiquem os estudos sobre os fenômenos psicossociais modificadores de comportamentos no Esporte. Exemplo disso, é que - Grupo de Estudos e Pesquisas em Psicossociologia do Esporte - "GEPPSE" coordenado pelo Prof.Dr. Antonio Carlos Simões, que estuda o Esporte e a Sociedade (forças sociais mantida entre o macro e o micro-sistema, espaço e urbanismo na prática e deteç̧ão de talentos esportivos; comportamento de liderança nos grupos esportivos; comportamento em relação ao grau de competitividade dentro dos grupos e a eficácia coletiva; relação entre coesão e atuação das equipes mediante o "Ideal próprio" dos técnicos e "Real equipe" dos atletas; validação de instrumentos de pesquisas que facilitem 0 levantamento de dados; dinâmica relacional humana que se manifestam às coisas, fatos $e$ impressões subjetivas; coerência orgânica e funcional na valorização das equipes esportivas, e a participação dos adultos na vida esportiva dos filhos.

Observa-se, então, que o "GEPPSE" estuda o comportamento humano no esporte como resultado de uma série de combinações de fatores incrivelmente ativos. O sistema de avaliação "ACS 1-2-3", desenvolvido por Simões (1999), se concentra nas orientações e contingências envolvidas as ações e conseqüências do comportamento ideológico de liderança implementado como "Ideal Próprio" pelos Técnicos frente ao "Real Equipe" dos Atletas com a eficiência e eficácia coletiva das equipes (Coesão social e de tarefas); indices de competitividade individual/coletiva na formação/organização das equipes e sistema sensorial preferencial. O "GEPPSE" mantém parceria com a Praendex/Arquitetura Humana, pelo Sistema "Predictive Index - PI" O sistema "PI" elabora perfis de personalidade ao analisar e cruzar as respostas de um instrumento de pesquisa que pode auxiliar as instituições/clubes/equipes a conhecerem profundamente seus componentes, o que possibilita a elaboração de planos de trabalhos em equipe, especialmente aqueles fatores ligados com a dominância, extroversão, paciência, ritmo, formalidade dos indivíduos, como também o moral da equipe, seu nível de energia e ritmo de trabalho conjunto. O sistema integrado "PI-ACS" pode definir o quadro estrutural e funcional das equipes esportivas, já que comprovam fatos que estão diretamente associados com os interesses e aspirações humanas no esporte. O Sistema integrado "PI-ACS" é desenvolvido sob a coordenação de Elmano Nigri, Neusa Miguel (Praendex/Arquitetura humana) e Antonio Carlos Simões a tudo relacionado com um alto grau de conhecimento às capacidades de desempenho dos indivíduos no esporte de "performance"

Paralelamente, o Grupo de Estudos e Pesquisas em Psicologia do Esporte - "GEPPE" 
coordenado pelo Prof.Dr. Dante De Rose Junior, estuda os aspectos psicológicos da competição infantil, aspectos cognitivos relacionados aos esportes coletivos; aspectos psicológicos do esporte de alto nível, especialmente o estresse e a ansiedade em sua relação com as atividades físicas e práticas esportivas de indivíduos não atletas. De Rose Junior (1996) desenvolveu a Lista de Sintomas de estresse Pré-competitivo infanto juvenil para detectar os principais sintomas de estresse em atletas de 10 a 17 anos. Esse estudo foi estendido a diversos esportes e já conta com um banco de dados com aproximadamente mil atletas, oferecendo condições de se traçar um perfil dos mesmos sob diferentes condições: idade, esporte e tempo de prática. Outra abordagem enfoca situações de estresse no esporte de alto nível e nas categorias menores - desenvolvido com aproximadamente 700 atletas de sete diferentes tipos de esportes, oferecendo a oportunidade de se compreender de forma mais específica as situações de estresse direta $\mathrm{e}$ indiretamente relacionadas à competição.

Os dois grupos se completam com a investigação psicossocial orientada em direção do sociológico e psicológico - sustentam que o mundo dos esportes estabelece relações altamente significativas - e atribui significados à realidade competitiva em que instituições, clubes, equipes e individuos se encontram. Enfim, a psicossociologia do Esporte representa uma disciplina básica aplicada - e que a Sociologia e a Psicologia são disciplinas entrelaçadas, e contribuem decisivamente nos estudos da problemática social e dos diversos tipos de comportamentos mantidos e projetados pelos dirigentes, técnicos e atletas no mundos dos esportes individuais e coletivos fenômenos psicossociais, no meio esportivo, nos clubes e nas equipes.

\section{REFERÊNCIAS BIBLIOGRÁFICAS}

ANTONELLI, F.; SALVINI, A. Psicologia del deporte. Roma, Editorial Minón, 1978.

BARREAU, J.J.; MORNE, J.J. Epistemologia y antropologia del deporte. Madrid, Alianza Editorial, 1991.

BERGER, P.I.; LUCKMAN, T. A construção social da realidade. Petrópolis, Vozes, 1985.

BRACHT, V. Esporte e poder. Brasília, Mineo, 1989.
CHAPPUIS, R.; THOMAS, R. L'équipe sportive. Paris, Presses Universitaries de France, 1988.

DELLA TORRES, M.B.L. O homem e a sociedade: uma introdução à sociologia. São Paulo, Editora Nacional, 1986.

HANIN, Y.L. Applying sport psychology: past, present and future. In: NADEAU, C.H. et alii. Psychology of motor behavior and sport. Champaign, Human Kinectics, 1979.

FREITAS, S.G. Racionalização e discriminação do esporte moderno. In: SIMPÓSIO ESPORTE: DIMENSÕES SOCIOLÓGICAS E POLÍTICAS. São Paulo, 1993. Anais. São Paulo, EEFE, 1993. p.26-9.

GONZÁLEZ, J.L. Psicología del deporte. Madrid, Editorial Biblioteca Nueva, 1997.

GUARESCHI, P.A.; JOVCHELOVITCH, S. Textos em representaçōes sociais. Petrópolis, Vozes, 1995.

HEINENAWN, K.; PREUSS, W. 25 years of the intemational review for the sociology of sport: a content analysis. International Review for the Sociology of Sport, v.25, n.1, p.3-17, 1990.

HEINILÃ, K. Impressions of the state, friends and prospects of the sociology of sport. International Review for the Sociology of Sport, v.25, n.1, p.339, 1990.

HELAL, R. O que é sociologia do esporte. São Paulo, Brasiliense, 1990.

MARTENS, R. About smocks and jocks. Journal of Sport Psychology, v.1, p.94-9, 1979.

MERLEAU-PONTY, M. O visível e o invisível. São Paulo, Editora Perspectiva, 1992.

MUCCHIELLI, R. O questionário na pesquisa psicossocial. São Paulo, Martins Fontes, 1978.

NICHOLSON, N. How hardwired is human behavior? Harvard Business Review, July/August, 1998.

PICHÒN-RIVIĖRE, E. Teoria do vínculo. São Paulo, Martins Fontes, 1988;

RIERA, J.; CRUZ, J. Psicología del deporte: aplicaciones y perspectivas. Barcelona, Ediciones Martínez Roca, 1991.

SALMELA, J.H. Psychology and sport: fear of applying. In: KLAVORA, P. et alii. Coach, athlete na sport psycholist. Toronto, University of Toronto, 1979.

SIMÕES, A.C. Sistema de avaliação "ACS - 1-2-3" São Paulo, LAPSE, 1999.

VUORI, I.; FENTEM, P. Salud. In: FUNCIÓN del deporte em la sociedad: salud, socialización, economia. Madrid, Ministério de Educación y Cultura/Consejo de Europa, 1996.

PEREZ, A.G: Filosofia del deporte. Madrid, Raiz Técnicas Gráficas, 1991.

ENDEREÇO: Antonio Carlos Simões
EEFEUSP

Av. Prof. Mello Moraes, 65

05508-900 - São Paulo - SP - BRASIL 